\title{
Davidia involucrata - ein dendrologischer Sonderfall
}

\author{
DiETER ZISSLER
}

\begin{abstract}
Davidia involucrata (Cornaceae) is a deciduous tree native to montane forests of western China and nowadays cultivated in most botanical gardens in Germany. Commonly it is known as handkerchief tree. Two varieties are differentiated: Davidia involucrata var. vilmoriniana and var. laeta. The genus name Davidia was based on the name of its discoverer, ARMAND DAVID (1826 - 1900), a French Jesuit missionary. Typical of the tree are its bracts, which turn from green to yellowish-white. These bracts attract pollinators (Hymenoptera, Coleoptera) and protect the flowers against rain.
\end{abstract}

\section{Zusammenfassung}

Davidia involucrata (Nyssaceae), nach ihrem Entdecker, dem französischen Jesuitenpater ARMAND DAVID (1826 - 1900) benannt, ist endemisch in den Bergwäldern im westlichen China. Heute wird sie in fast allen deutschen botanischen Gärten kultiviert. Bei uns ist sie als Taschentuch- oder Taubenbaum bekannt. Zwei Varietäten werden unterschieden: Davidia involucrata var. vilmoriniana und var. laeta. Der Baum ist besonders durch seine Brakteen gekennzeichnet, die im Laufe der Blütenentwicklung einen Farbwechsel von grün nach gelblich-weiß durchlaufen, wobei sie die Fähigkeit zur Photosynthese verlieren. Die Brakteen werden im Zusammenhang mit der Anlockung von Bestäubern (Hymenoptera, Coleoptera) und als Schutzeinrichtung gegen Regen gesehen.

\section{Systematische Einordnung und Namensgebung}

Ein hinsichtlich seines Erscheinungsbildes unauffälliges, aufgrund seiner Blütenstände jedoch außergewöhnliches Ziergehölz europäischer Garten- und Parkanlagen ist Davidia involucrata, ein Tupelobaumgewächs (Nyssaceae) aus der Ordnung der Hartriegelartigen (Cornales). Von manchen Autoren wird der Taschentuchbaum aber auch in die eigene Familie Davidiaceae gestellt. Es gibt nur eine Art dieser Gattung, die vom Franzosen BAILlON als Davidia involucrata beschrieben wurde. Aufgrund unterschiedlicher Ausbildung von Zweigen, Blättern und Früchten werden heute wenigstens zwei Varietäten unterschieden, nämlich var. vilmoriniana und var. laeta. Die am häufigsten kultivierte Varietät ist offenbar var. vilmoriniana, die in fast allen deutschen Botanischen Gärten vorkommt. COCKER (1952) und IvENs (1953) betrachten Davidia involucrata var. vilmoriniana aufgrund ihrer Früchte sogar als eigene Art.

Der Gattungsname Davidia wurde zu Ehren ihres Entdeckers, des französischen Jesuitenpaters ARMAND DAVID (1826-1900) benannt. Ab 1861 bereiste dieser als Missionar für mehrere Jahre China und wurde dabei zu einem der namhaftesten Wegbereiter allseitiger Kenntnis der ostasiatischen Pflanzen- und Tierwelt. In der
Literatur wird er häufig als Abbé DAVID oder auch Pére DAVID genannt. ARMAND DAVID hat eine große Zahl von Pflanzen und Tieren entdeckt. Nicht wenige Bäume und andere Blütenpflanzen und unter den Insekten besonders Schmetterlinge sind nach ihm benannt. Der Milu (Elaphurus davidianus) trägt im Deutschen den Namen Davidshirsch. Er weist zwar Merkmale von Hirsch, Rentier, Rind und Esel auf, lässt sich aber mit Rothirschen kreuzen. Diesen, der vermutlich schon lange vor unserer Zeitrechnung in freier Wildbahn ausgestorben war, entdeckte DAVID 1865 in einem Park bei Peking und schuf so die Voraussetzungen für ein umfangreiches Zuchtprogramm, das den Milu vor dem vollständigen Aussterben bewahrte.

Der Art-Beiname involucrata nimmt Bezug auf die beiden Hochblätter, die gleich einem Hüllkelch das unmittelbare Umfeld eines jeden Blütenstandes begrenzen (Abb. 1, 2) und so den Baum von Mai bis Juni schmücken (s. u.). Blühende Davidien sind meist mindestens 20 Jahre alt.

\section{Verbreitung und Winterhärte}

Davidia involucrata ist in den Bergwäldern im westlichen China endemisch (FANG \& CHANG 1983). Sie ist bei uns winterhart. Von der Insel Mainau im Bodensee wird berichtet (PFINDEL 


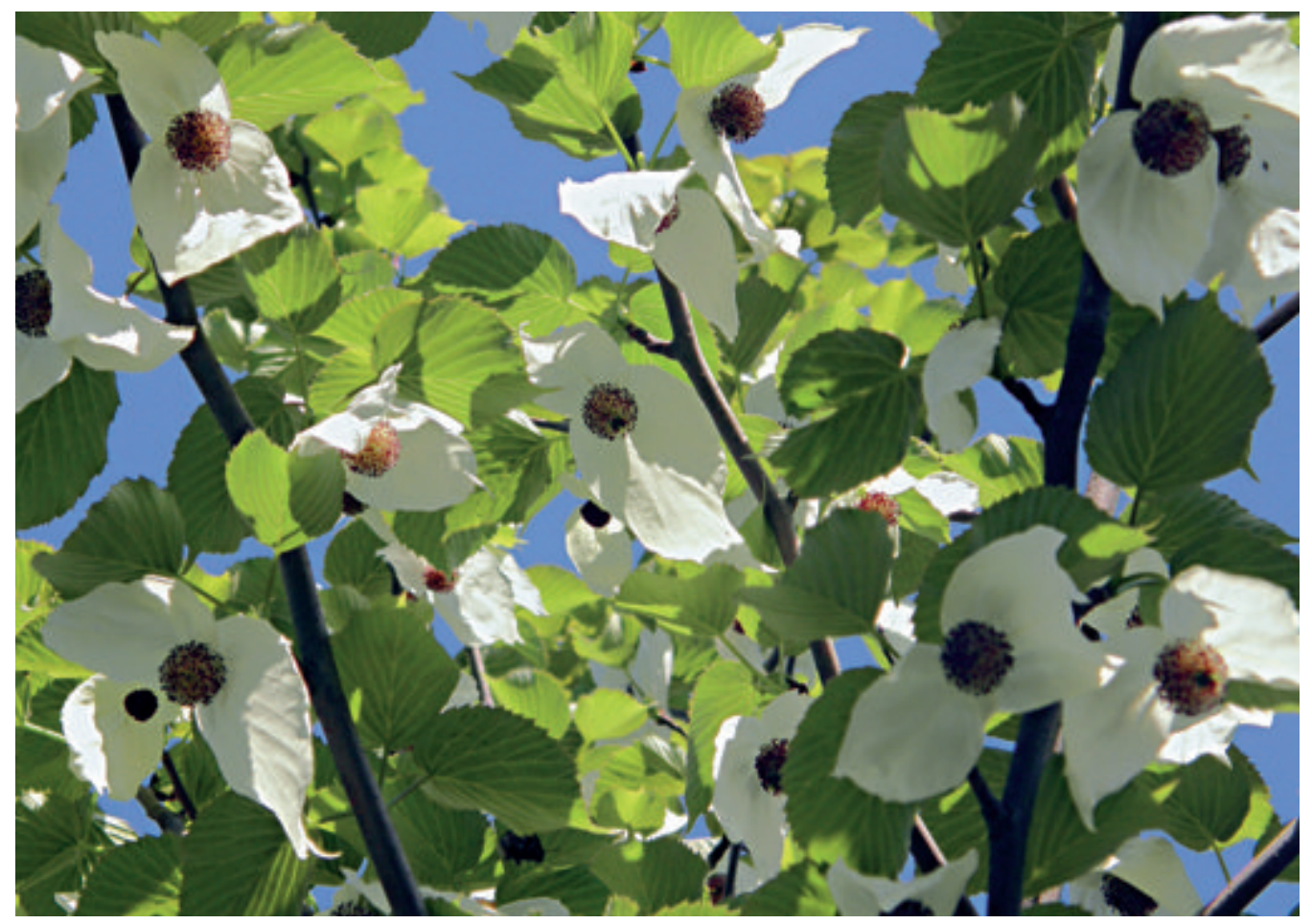

\& MEYer 2005), dass das älteste Exemplar im Arboretum der Insel „wohlgeschützt zwischen einer Zeder und einer Buche" die Frostperiode 1955 bei $-20^{\circ} \mathrm{C}$ unbeschadet überstanden hat. Auch den Extremwinter 2008/9 haben die meisten Davidien wohl gut überlebt.

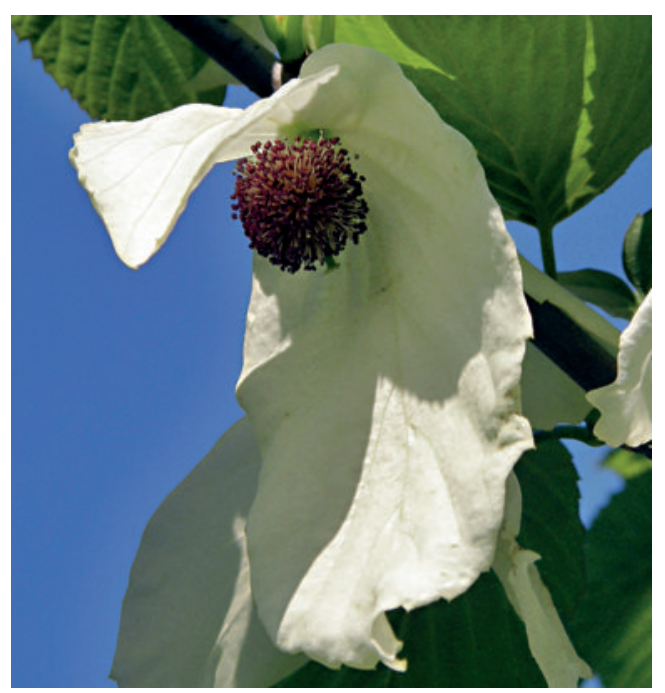

\section{Wuchsform}

Davidia ist ein Laubbaum von annähernd kegelartigem Wuchs und kann in ihrer Heimat eine Höhe von 20 m erreichen, bleibt aber meist kleiner, insbesondere in Kultur. Ihre herzförmigen, im allgemeinen bis $15 \mathrm{~cm}$ langen und bis $8 \mathrm{~cm}$ breiten Blätter färben sich im Herbst gelblich-braun und fallen dann ab. Aufgrund der Wuchs- sowie Blattform wird der Taschentuchbaum im vegetativen Zustand nicht selten mit einer Linde verwechselt (KRÜSSMANN 1960). Die Früchte bleiben noch einige Zeit länger als die Blätter am Baum (Abb. 3). Sie sind langgestielt, elliptisch, 3-4 cm lang und etwa $2,5 \mathrm{~cm}$ breit. Ihre Oberfläche ist längsgefurcht, lederbraun und hell punktiert. Es handelt sich um 3-5-samige Steinfrüchte, die äußerlich etwas an Walnüsse erinnern.

Abb. 1 (oben): Davidia involucrata var. vilmoriniana; rote Blütenstände, umgeben von zwei gelblichen Brakteen.

Abb. 2 (links): Davidia involucrata var. vilmoriniana; die gelblichweißen Brakteen locken bestäubende Insekten (Apidae und Coleoptera) an und schützen die rötlichen Antheren vor Regen. 


\section{Blüten und Blütenstände}

Die Blütenstände sind kugelige Köpfchen, in denen sich zahlreiche männliche Blüten mit rötlichen Staubblättern pinselartig um eine zentrale weibliche Blüte mit hellgrünlichem Stempel ordnen. Eine Blütenhülle aus Kelch- und Kronblättern ist nicht ausgebildet. Blütenstand und Hochblätter erwecken bestäubungsbiologisch den Eindruck einer Einheit, weshalb man hier von einem Pseudanthium spricht (BURR \& BARTHLOTT 1993). Die beiden gegenständigen, unterschiedlich langen (bis fast $20 \mathrm{~cm}$ ) Brakteen sind anfangs grün, dann gelb und zuletzt cremeweiß gefärbt (Abb. 1, 2). Die cremeweißen papierartigen Hochblätter sind ebenso zart wie windempfindlich, so dass man nicht nur nach Ende der Blühperiode, sondern auch an windigen Tagen den Boden unter den Davidien so vorfinden kann, als hätte es Papiertaschentücher geregnet (Abb. 5, vgl. deutschen Volksnamen). Im Englischen heißt die Davidie auch handkerchief tree. "Als große schwebende Schmetterlinge“ glaubt der als „Doyen der modernen Sammler am Rande der Welt" in die Geschichte der Pflanzenjäger eingegangene ERNEST HENRY WILSON die Hochblätter sehen zu wollen (WHITTLE 1971). Dass man beim Anblick der von den Brakteen erfüllten Baumkrone auch an Vögel, gar an Tauben, denken kann, führte zum deutschen Namen Taubenbaum, im Englischen dove tree.

Aufgrund der Hochblätter wird Davidia als dendrologischer Sonderfall betrachtet. Diese enthalten ebenso wie die Laubblätter und die Antheren verschiedene Flavonoide (Hu et al. 2007), deren Funktion diskutiert wurde (z. B. BURR \& BARTHLOTT 1993), aber letztlich unbekannt blieb. BURR \& BARTHLOTT (1993) haben die UV-Absorption der cremefarbenen Brakteen nachgewiesen, zudem aber auch gezeigt, dass die Antheren stärker UV-absorbierend sind als die Hochblätter. Sie deuten deshalb DAVIDIA als ein entomophiles Gewächs.

Diese Entomophilie wurde kürzlich von einer chinesisch-deutschen Forschungsgruppe (JI-FAN

Abb. 3 (oben): Früchte des Taschentuchbaumes.

Abb. 4 (unten): Frucht im Detail.
SUN et al. 2008) genauer untersucht. Die nektarfreien Köpfchen werden im Wesentlichen von Pollen sammelnden Bienen der Gattungen Apis, Xylocopa, Halictus und Lasioglossum sowie von Käfern aus den Familien der Elateridae, Cetoniidae und Nitidulidae besucht.

In Experimenten mit künstlichen Hochblättern aus grünen und weißen Papieren, die in Größe und Form den natürlichen Brakteen ähnelten, konnte gezeigt werden, dass die Hochblätter neben dem Anlocken der Insekten noch eine zweite Aufgabe erfüllen, die die Bezeichnung Schauapparat nicht einschließt. Die Brakteen schützen nach Art eines Regenschirms den Blütenstand vor Regenwasser und verhindern so, dass der Pollen ausgewaschen wird und verloren
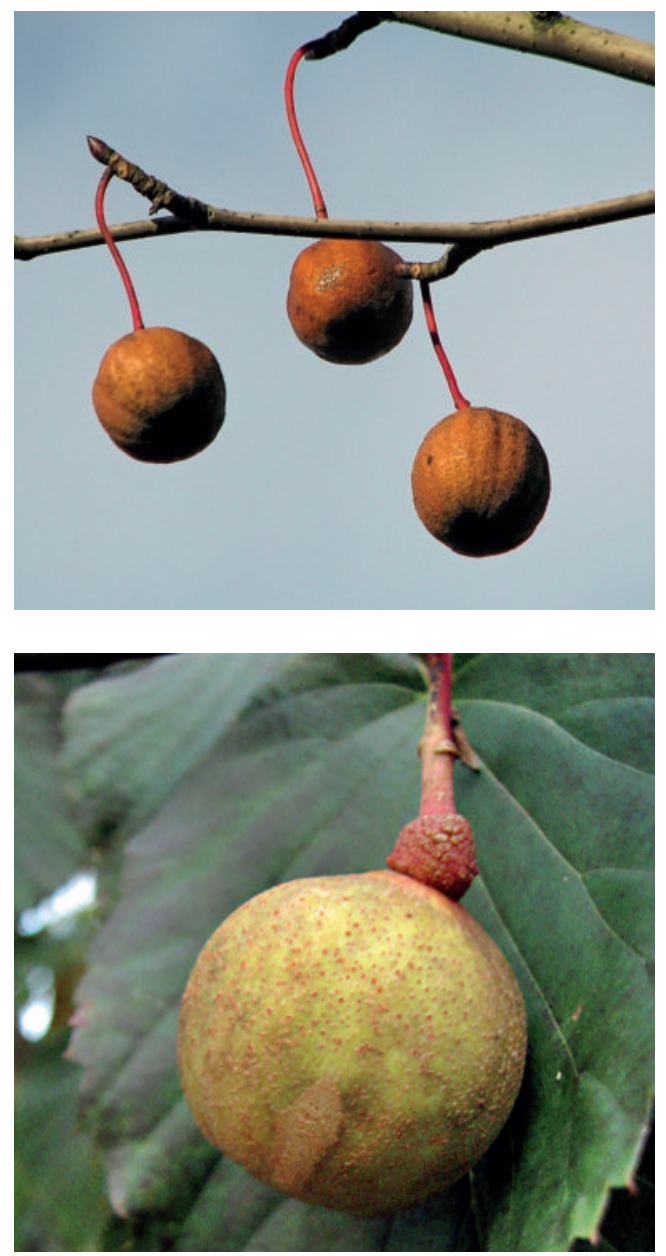


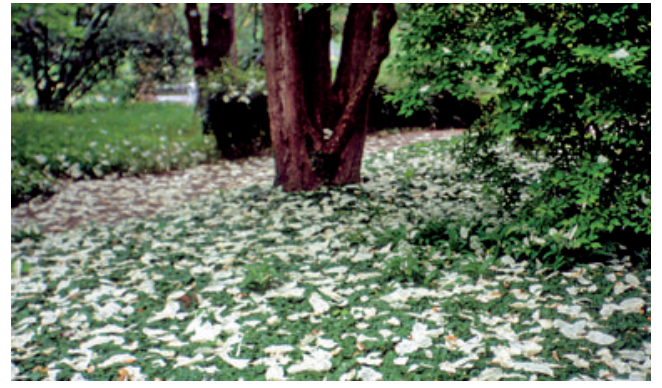

geht. Damit wird verständlich, dass Davidia während der Regenzeit blühen kann, ohne dabei Schaden zu nehmen.

\section{Taschentuchbäume im}

\section{Botanischen Garten Freiburg}

Beide Varietäten hat der Botanische Garten der Universität Freiburg i. Br. über Jahrzehnte kultivieren können. Das mag vielleicht auch darauf zurückzuführen sein, dass FRIEDRICH OEHLKERS (1890-1971), ein früherer Freiburger Ordinarius für Botanik und Direktor des Gartens, die Davidia zu seinem Lieblingsbaum auserkoren hatte (VOGELLEHNER 2001).

OEHLKERS war nicht nur unter Botanikern geachtet und geschätzt. Er galt auch in Freiburg, ja in Baden, als Persönlichkeit von seltenem Format und ungewöhnlicher Integrität. Es lag geradezu auf der Hand, dass er der erste Rektor aus der $\mathrm{Na}$ turwissenschaftlichen Fakultät der Universität Freiburg nach dem 2. Weltkrieg wurde (BOPP 1991). OehlKers' Geburtstag war der 6. Mai. Man darf daran erinnern, dass in jedem Frühjahr Oenlkers' Mitarbeiter, Schüler und vor allem die Gärtner bestrebt waren, ihm zum Geburtstag einen Davidien-Zweig mit Blüten auf den Tisch zu stellen. Nicht selten waren die Gärtner des Botanischen Gartens schon Anfang April geradezu nervös, ob denn der Baum rechtzeitig blühe.

\section{Dank}

Mein Dank gilt Herrn Professor Dr. THOMAS SPeCK, Direktor des Botanischen Gartens der Universität Freiburg, für die Anregung und Ermunterung, diese Erinnerungen aufzuschreiben.
VERONIKA WAEHNERT vom Botanischen Garten Freiburg und JULIA SCHWARZ, Bibliothekarin, danke ich ebenfalls für hilfreiche Recherchen. Großer Dank für Hilfe bei englischen Übersetzungen und die Aufarbeitung des Manuskriptes gilt auch Dr. Randall Cassada, Birgit Büsch, Martin EIBNer und STEFAn Heyl. Besonders herzlich danke ich Elfriede BaUmanN, der langjährigen Mitarbeiterin von Professor OeHLKERs, die mir vielfach bei der Erstellung dieses Beitrages behilflich war.

\section{Literatur}

Bopp, M. 1991: Friedrich OeHLKERS, Forscher und Lehrer. - Freiburger Universitätsblätter 111: 69 - 75. BuRR, B. \& BARTHLOTT, W. 1993: Untersuchungen zur Ultraviolettreflexion von Angiospermenblüten II. Magnoliidae, Ranunculidae, Hamamelididae, Caryophyllidae, Rosidae. - Trop.-Subtrop. Pflanzenwelt 87, Akad. Wiss. Lit. Mainz: 1-193.

Cocker, H. 1952: Davidias. - The Gardeners' Cronicle. Vol. CXXXII: 226.

FAnG, W. P. \& Chang, C. Y. 1983: Flora Republicae Popularis Sinicae. Vol. 52. - Beijing.

HARTE, C. 1994: Oenothera - Contributions of a plant to biology. - Berlin u. a.

Hu. J. Y., ZHANG, S. L. Su, Z. X. \& LiAO, Y. M. 2007:

Pollinator attraction by Davidia involucrata, I. Color.

J. Plant Ecol. 31: 166-171.

IvENS, F. L. S. 1953: Davidia: Are there two species. -

Gardeners' Chronicle, Vol. 133: 22.

KRÜssmanN, G. 1960: Handbuch der Laubgehölze.

Band I. -Berlin, Hamburg.

Marquardt, H. 1974: Friedrich OehlKers 1890 -1971. -

Ber. Deutsch. Bot. Ges. 87: 185 - 192.

Oehlekers, F. 1956: Das Leben der Gewächse.

Ein Lehrbuch der Botanik. Erster Band. Die Pflanze als Individuum. - Berlin, Göttingen, Heidelberg. Pfindel, J. \& Meier, H. - D. 2005: Die Pflanzenwelt der Mainau. Ein botanischer Führer durch Park und Gärten der Insel. - Stuttgart.

Sun, J.-F., Gong, Y.-B., Renner, S. S. \&

HuANG, S. Q. 2008: Multifunctional bracts in the dove tree Davidia involucrata (Nyssaceae: Cornales): Rain protection and pollinator attraction. -

Amer. Naturalist 171: 119 - 124 .

VogelleHner, D. 2001: Dreieinhalb Jahrzehnte Forschung, Lehre, Botanischer Garten. Ein Rückblick. Anhang: Schriftenverzeichnis und betreute wissenschaftliche Arbeiten. - Württ. bad. Landesver.

Naturkunde und Naturschutz N. F. 17: 717 - 744.

Whittle, T. 1971: Pflanzenjäger. Die abenteuerliche Suche nach dem Grünen Gold. - München.

Abb. 5: Davidia involucrata var. vilmoriniana; die am Ende der Blühperiode abgefallenen Brakteen bedecken den Boden. 